\title{
Reliability and Validity of a New Fitness Intermittent Judo Test
}

\author{
Mohamed Saifeddin Fessi ${ }^{1,}{ }^{*}$, Saber Nouira ${ }^{1}$, Faical Farhat ${ }^{1}$, Hamdi Chtourou ${ }^{1}$, Said Ahmaidi ${ }^{2}$ and \\ Wassim Moalla ${ }^{1}$ \\ ${ }^{1}$ UR 15JS01 EM2S: Education, Motricity, Sport and Health, High Institute of Sport and Physical Education, Sfax University, Sfax, Tunisia \\ ${ }^{2}$ Physiological Adaptations to Exercise and Effort Rehabilitation, University of Picardie Jules-Verne, Amiens, France \\ "Corresponding author: UR 15JS01 EM2S: Education, Motricity, Sport and Health, High Institute of Sport and Physical Education, Sfax University, Sfax, Tunisia. Tel: +216-55575576, \\ E-mail: saifelfessi@gmail.com
}

Received 2018 August 16; Accepted 2018 August 29.

\begin{abstract}
Objectives: The aim of this study was to assess the validity and the reliability of a new fitness intermittent judo test using the basic movement patterns of judo.

Methods: Forty-one national level judo male athletes participated in this study. Eighteen judokas performed the intermittent judo fitness test consisting of $10 \times 30 \mathrm{~s}$ maximal interspersed with 15 second rest, the Australian shuttle test, the special judo fitness test and a judo fight. Twenty three judokas performed twice the intermittent judo fitness test on different days.

Results: The results showed significant correlations between the biggest number of Uchi-komi during the intermittent judo fitness test $\left(\right.$ Uchi-komi $\left.{ }^{\text {best }}\right)$, the longest distance covered during Australian shuttle test and the total projection in the special judo fitness test $(\mathrm{P}<0.01)$. Likewise, a significant relationship was demonstrated between the total Uchi-komi performed in the new judo test (Uchi$\left.k o m i^{\text {Tot }}\right)$ and both the total distance covered during Australian test and the total projection during the special judo fitness test $(\mathrm{P}<$ 0.01). In addition, a significant correlation of the rating of perceived exertion was observed between the intermittent judo fitness test and judo fight $(\mathrm{P}<0.05)$. The intra-class correlation coefficient analysis revealed relatively strong test-retest reliability for Uchi$k o m i^{\text {best }}$ and $U c h i-k o m i{ }^{\text {Tot }}(>0.90)$. The smallest worthwhile change showed that a change of 2 and $4 U c h i-k o m i$ in $U c h i-k o m i^{\text {best }}$ and Uchi-komi ${ }^{\text {Tot }}$ respectively is occurred.

Conclusions: The new fitness intermittent judo test is both valid and reliable measurement tool to assess judokas' fitness at the basic judo movement patterns.
\end{abstract}

Keywords: Fitness Testing, Reliability, Validity, Uchi-komi, Judo Athletes

\section{Background}

Time-motion analysis in judo athletes highlights that judo is a complex sport with random movement patterns and repetitive maximal or sub-maximal short duration effort (20 - $40 \mathrm{~s}$ ) interspersed by interval breaks of $10 \mathrm{~s}(1,2)$. Usually, two positions have been described as fight components: (i) the standing work (Nage-waza) and (ii) the ground work (Ne-waza). Nage-waza is characterized by the isometric effort of the upper limb using static force and dynamic effort and by the explosive effort at the lower extremity in the use of pseudo-dynamic force (3). However, Ne-waza, consists in immobilizing or strangulating the opponent or impairing their elbow joint. These efforts are mostly isometric and dependent on pseudo-dynamic power to maintain a posture or control the opponent (4-6).

Previous studies had assessed the fitness of judokas through treadmill or cycle ergometer exercises $(7,8)$. Such laboratory tests were too general and they were not judospecific since they do not take into account the particular motor skills and movement patterns performed during a judo competition (8-10). Few studies have been supporting the concept of the specificity in assessing the physical fitness of judokas. In this context, Thomas et al. (11) adapted the Leger-Mercier-test in judo with the reduction of the 20-m distance to 15 - $\mathrm{m}$ and the introduction of a projection in achieving the shuttle. Sterkowicz (12) developed the special judo fitness test (SJFT) which has been used in different judo investigations $(8,13)$. These researches have reported significant relationships between SJFT and both physiological and physical parameters of judokas and have also established classification criteria of judoka according to measured variables during this test (8). Almansba et al. (9) validated the Uchi-komi fitness test as an intermittent test performed in six sets lasting $23 \mathrm{~s}$, which increase by 3 $\mathrm{s}$ and separated by $4 \mathrm{~s}$ of rest that increase by $2 \mathrm{~s}$ per set. However, the previous tests included running in their protocols. Thus, in some cases, the judoka can use additional energy in running with strong peripheral fatigue in lower 
limbs, differently from what happens in combats, when upper limb muscles are the most demanded (8). In addition, Azevedo et al. (14) proposed an endurance-specific test for judokas, based on the Uchi-komi (drill of techniques without projection) to evaluate the aerobic capacity of judokas using the lowest blood lactate concentration. Furthermore, Del Vecchio et al. (15) assessing the physical fitness of judokas through the all-out Uchi-komi performed in 20, 30 and 40 s. Moreover, Santos et al. (10) proposed the Santos test in uses specific actions and conditions of judo to determine the aerobic-anaerobic transition zone $(7,10)$ Different technical skills were performed during the Santos test in successive sequences, which are close to the intermittent nature of judo fights. However, the sequence of specific movements is not identical to actual judo fights despite their use of the basic movement patterns. Tavra et al. (16) analysed the discriminant and factorial validity of four specific judo tests (SJFT, Uchi-komi fitness test, Santos test and specific station judo ability). They suggests that all these four specific tests have a similar objective measurement and that the SJFT and the Uchi-komi fitness test better discriminated the elite from the subelite female judokas (16). Thus, different intermittent protocol based in Uchi-komi approach, with different manipulation ratios of effort and rest $(17,18)$ were used to investigate the fitness of judokas, suggesting that these exercises could elicit similar physiological demands to those observed in judo fighting (19). Taking all these parameters into account, the challenge was to develop a more appropriate test which is closely linked to the construct of judo fights. The aims of this study were, therefore, to determine the reliability of a new fitness intermittent judo test (FIJT) with basic movement pattern, and to assess the components validity of the FIJT.

\section{Methods}

\subsection{Participants}

Forty-one national level judo male athletes (age: 21.5 \pm 1.4 years; body mass: $78.2 \pm 11.3 \mathrm{~kg}$; height: $177.0 \pm 7.8$ $\mathrm{cm}$; body fat: $13.1 \pm 4.5 \%$ ) voluntarily participated in this study. The consent form was signed prior to beginning the study. Each participant had at least 12 years of judo training experience. Participants performed an average of 15 h.wk $\mathrm{k}^{-1}$ of regular judo training in their team club and engaged in competition schedule such as the national championships at different weight categories ranging from halflight-weight (-66 kg) to middle-weight (-90 kg). The study was approved by the Local Ethics Committee in accordance with the principals outlined in the declaration of Helsinki.

\subsection{Procedures}

The component validity and the reliability of the new developed FIJT were assessed in two parts. First, we estab- lished the relationship between the FIJT and both the shuttle Australian test and the SJFT. All tests were randomly performed on separate days in 18 judo athletes. The ratings of perceived exertion (RPE) were assessed during both the FIJT and the judo fight to check the similarity of psychological and physiological stress induced by the FIJT and that of the judo fight. Second, we examined the reliability of the FIJT in 23 judokas who completed the FIJT twice separated by at least 72-hours interval including a technical and tactical training session the day after the test and no training took place 24-hours prior to testing to minimize the effects of fatigue. To avoid the effect of diurnal variations, the two FIJT were completed at the same time of the day (between 3:00 P.M. and 5:00 P.M.). Participants who performed the test (i.e. Tori, the attackers) conducted the two FIJT with the same partners (i.e. Uke, the defenders) who were from the same weight category of Tori. Tori used the same technique in projection, reversal in ground and Uchi-komi during both test and retest. For all tests performed, participants were familiarized with test protocols one week before data collection. All testing sessions were performed on judo mat (Tatami) after 15 min of standard warm-up including jogging and dynamic stretching followed by 5 min of rest. For specific tests, participants finished warm-up by Uchi-komi and Nage-komi.

\subsection{Fitness Intermittent Judo Test}

The new developed FIJT design was inspired by the analysis of judo practice. The total test time was $7 \mathrm{~min}$ and $30 \mathrm{~s}$. It consists of 10 maximal $30 \mathrm{~s}$ periods of efforts interspersed with a $15 \mathrm{~s}$ rest period. Each period of effort reproduced the accurate basic movement pattern of judo including, gripping (Kumi-kata), displacement by pulling (Ayumi), projection (Nage-komi), and groundwork (Ne-waza), and was completed by the Uchi-komi which is recognized as a fundamental training exercise in judo. As for the test performed on Tatami, a distance of $4 \mathrm{~m}$ indicated by markers separates between the starting point and the ending point.

Before the beginning of each period, Tori gripped the Uke by Kumi-kata and pulled him overall $4 \mathrm{~m}$ with Ayumi displacement. Immediately, Tori projected Uke twice by simultaneous Nage-komi. After the second projection, Uke was on all fours on the ground Tori was conducting a reversal Ne-waza to immobilize Uke. Finally, Tori and Uke were in standing position and Tori performed Uchi-komi until the end of the time (30 s). During the rest period, Tori and Uke reached the next start and were waiting for the next audio signal. The number of performed Uchi-komi was calculated for each period, and considered as a dependent variable.

The performance FIJT indexes were: the best Uchi-komi (Uchi-komi ${ }^{\text {best }}$ ) and the total Uchi-komi (Uchi-komi $\left.{ }^{\text {Tot }}\right)$ where Uchi-komi $i^{\text {best }}$ was the best number of Uchi-komi during ten 
periods, then Uchi-komi ${ }^{\text {Tot }}$ was the sum Uchi-komi of the ten periods. The investigator explained in details the conditions of the test sequence for the Tori and the Uke. Only the fully performed Uchi-komi, including both the imbalance (Kuzushi) of the Uke and the correct placement of the body (Tsukuri) of the Tori, was taken into account. Tori used the same technique, according to his choice, in Kumi-kata, Nage-komi, Ne-waza, and Uchi-komi during the ten periods of the FIJT. When Tori used a different or not full technique, the investigator alert Tori and does not consider this trial in calculated FIJT performance indexes.

\subsection{Shuttle Australian Test}

Participants performed the shuttle running Australian test $(20,21)$ which consisted of running back and forth 5 , 10,15 and $20 \mathrm{~m}$ distances to cover the longest distance possible in $30 \mathrm{~s}$ for six times, interspersed with $35 \mathrm{~s}$ of active recovery. The longest distance covered in $30 \mathrm{~s}$ allows an appreciation of the anaerobic metabolism power and the total distance indicated the anaerobic metabolism capacity of participants.

\subsection{Special Judo Fitness Test}

All participants completed the SJFT (12) which consisted in projecting twice Uke placed at $6 \mathrm{~m}$ from each other using the Ippon-seoi-nage during three periods of 15, 30 and $30 \mathrm{~s}$ interspersed by recovery intervals of $10 \mathrm{~s}$.

\subsection{Judo Fight}

The RPE of the judo fight was taken from all participants involved in a fight according to the regulatory standards. Each fight was refereed by three judges and the official rules were adopted. Nevertheless, even a projection that can induce the victory did not interrupt the fight. The fight ends on reaching the 5 min effective time and the winner is the judoka who make more collect points.

\subsection{Rating of Perceived Exertion During FIJT and Judo Fight}

Psychological and physiological exercise perception was quantified using a modified 10-point Borg scale (22). Each Tori was responded to the question: "How did you perceive your exertion during the test or the fight?" $30 \mathrm{~min}$ after the FIJT and/or judo fight to record a subjective estimation of the physiological load of the test or/and the fight.

\subsection{Data Analysis}

Data are presented as means \pm SD. One-way ANOVA followed Tukey post-hoc were used to explore differences in performed Uchi-komi during the FIJT. Pearson's product moment correlation coefficients were used to examine the associations between the performance of the FIJT and other parameters, and between RPE during the FIJT and the judo fight. The magnitudes of the correlations (23) were considered as trivial $(\mathrm{r} \leq 0.1)$, small $(\mathrm{r}>0.1-0.3)$, moderate ( $r>0.3-0.5)$, large ( $r>0.5-0.7)$, very large ( $r>0.7-0.9)$, nearly perfect $(r>0.9)$, and perfect $(r=1)$. Systematic bias was investigated using a dependent $t$-test to explore mean performance differences between tests and retests sample of the FIJT. The effect size (ES) was calculated from the ratio of the mean difference in the pooled standard deviation to assess meaningfulness of differences between test and retest FIJT performance. The magnitude of ES was classified as trivial $(\leq 0.2)$, small $(>0.2-0.6)$, moderate $(>0.6$ $-1.2)$, large $(>1.2-2.0)$ and very large $(>2.0)$ based on Batterham and Hopkins guidelines (24). To determine the relative reliability of the FIJT, an average measure of the intraclass correlation coefficient (ICC) with alpha analysis was calculated (25). In order to test the absolute reliability of the FIJT, the standard error of measurement (SEM) was established to reflect within-subject reproducibility for the measures outlined above (25). To establish the usefulness of the FIJT, the smallest worthwhile change (SWC) was determined $(25,26)$. All statistical analyzes were conducted using the statistical package for the social sciences (SPSS version 18.0, SPSS Inc, Chicago, IL, USA). The level of significance was set at 0.05 .

\section{Results}

The analysis of the number of Uchi-komi during the FIJT showed significant differences between the ten periods of the test $(\mathrm{P}<0.01$; Figure 1$)$. We observed a higher number of Uchi-komi performed in the first period compared to seconds period $(\mathrm{P}<0.05)$. Moreover, we showed a significant decline in the number of Uchi-komi from the second to the 6 th period $(\mathrm{P}<0.05)$ with no difference between sixth and seventh periods. Afterwards, a new decline in Uchi-komi number from 7 th to 9 th periods was observed. Finally, we observed a greater number of Uchi-komi in the last period with a higher value than those observed through the 5th, 6 th, 7 th, 8 th and 9 th periods $(\mathrm{P}<0.05)$ and similar to those observed in the 4 th $(16.12 \pm 2.43$ vs. $16.14 \pm 1.31 ; \mathrm{P}>0.05)$.

The mean $( \pm S D)$ of performance done during the different tests performed in the first part of this study are given in Table 1. A significant relationship was observed between the Uchi-komi ${ }^{\text {best }}$ and Uchi-komi ${ }^{\text {Tot }}$ performed in the FIJT and the longest and the total distance covered in Australian test, the number of projections during the SJFT, and the RPE of judo fight (Figure 2).

The test-retest of Uchi-komi $i^{\text {best }}$,Uchi-komi ${ }^{\text {Tot }}$, the ICCs, the ES, SEMs, and SWCs occasioned between the two tests are given in Table 2 . No difference was found for the Uchikomi $^{\text {best }}(\mathrm{P}=0.13)$ and $U$ chi-komi ${ }^{\mathrm{Tot}}(\mathrm{P}=0.07)$ during the two FIJT, with an ICCs higher than 0.9 for both Uchi-komi $i^{\text {best }}$ and 


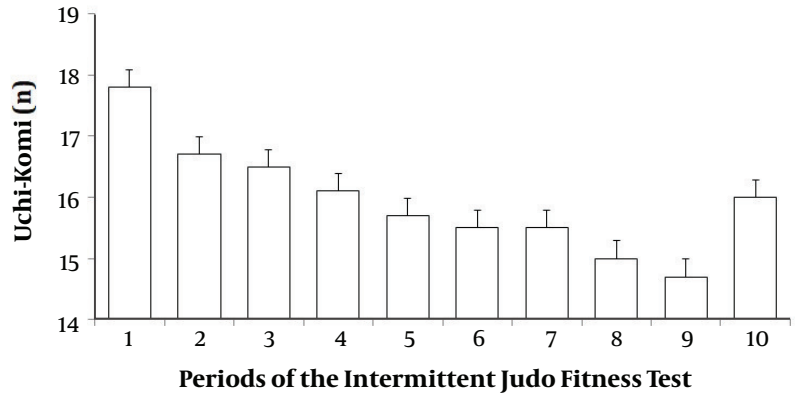

Figure 1. Kinetic of Uchi-komi performed during the fitness intermittent judo test

Tables 1. Mean \pm SD of Uchi-komi During the Fitness Intermittent Judo Test, the Running Distance During the Shuttle Australian Test, the Number of Projections in the Special Judo Fitness Test and the Rating of Perceived Exertion of the Fitness Intermittent Judo Test and Judo

\begin{tabular}{|cc|}
\hline Fitness Intermittent Judo Test & Values (Mean \pm SD) \\
\hline${\text { Uchi-komi } i^{\text {best }} \text { (No.) }}$ (Nohi-komi ${ }^{\text {Tot }}$ (No.) & $17.82 \pm 1.56$ \\
\hline Shuttle Australian test & $159.67 \pm 13.63$ \\
\hline Longest distance in 30s (m) & $138.13 \pm 5,36$ \\
\hline Total distance in test (m) & $767.45 \pm 21.82$ \\
\hline Projections in special judo fitness test & \\
\hline Period 1(No.) & $6.15 \pm 0.86$ \\
\hline Period 2 (No.) & $11.23 \pm 1.26$ \\
\hline Period 3 (No.) & $11.13 \pm 0.95$ \\
\hline Total projections (No.) & $28.43 \pm 2.66$ \\
\hline Rating of perceived exertion & $6.56 \pm 1.39$ \\
\hline Fitness intermittent judo test & $5.88 \pm 1.16$ \\
\hline Judo fight & \\
\hline
\end{tabular}

$U c h i-k o m i^{\text {Tot }}$. The SWC was 2.01 and 2.96 for Uchi-komi $i^{\text {best }}$ and $U$ chi-komi $i^{\mathrm{Tot}}$, respectively.

\section{Discussion}

The aim of the present study was to assess the validity and reliability of a new intermittent test with a judospecific movement pattern. The present data showed that FIJT could be a reliable and useful evaluation of judokas' ability to repeat intermittent efforts with a basic fundamental movement pattern in judo fights. The results of the present study showed a bigger number of Uchi-komi performed in the first period that could be attributed in part to the physical freshness in the beginning of the effort during the test. However, the decrease in the number of Uchikomi from the second to the ninth period can be explained by the gradual onset of fatigue that is usually observed during general and specific intermittent protocol. In this con- text, it has been previously demonstrated that the intermittent Uchi-komi protocol resulted in high glycolytic demand (18). The lack of biological measurements such lactate is considered as a major limitation of the present investigation. Increases in Uchi-komi during the last period of the FIJT could be the result of energy supply exported by the phosphagens pathway and oxidative phosphorylation associated with subject's motivation it being the end of the test.

In the current FIJT, the effort to pause ratio (2:1) is associated with a general decline in the number of Uchi-komi. In related performance kinetic during the FIJT to previous analyses of protocol used intermittent Uchi-komi exercise, Baudry and Roux (17) observed that an effort to rest ratio of both 1:3 and 1:5 resulted in a higher number of Uchikomi with more performance stability compared to 1:1 effort to rest ratio during $6 \times 40 \mathrm{~s}$ protocol. Franchini et al. (18) investigated intermittent Uchi-komi protocol using the 1:1 effort to rest ratio and changing the duration of the effort $(6 \times 30 \mathrm{~s}, 9 \times 20 \mathrm{~s}$ and $18 \times 10 \mathrm{~s}$ with 3 different judo techniques (O-uchi-gari, Harai-goshi and Seoi-nage) observed that the athletes were able to perform the greatest number of Uchi-komi with O-uchi-gari, but no effect of Uchikomi protocol duration was observed. This suggests that the effort to rest ratio is the most important variable to be manipulated to elicit performance in intermittent Uchikomi protocol $(18,19)$. Nevertheless, the increased or decreased performance observed during the present FIJT is in agreement with the kinetic of attack frequency, performed during a judo fight, previously reported $(1,2)$.

The present data revealed that the value of RPE after the FIJT was similar to that observed after judo fights (6) where the RPE was correlated to lactate' measurements. The significant correlation found in the current study between the RPE of FIJT and judo fight confirmed that the FIJT reproduced a similar physiological and psychological effort during judo fights, which is the major aim of this new test. In fact, the FIJT development was closely linked to the construct of judo fights. Moreover, we found that the number of Uchi-komi performed during the first period of the FIJT (best performance during the test; Uchi-komi ${ }^{\text {best }}$ ) was almost perfectly significant with the number of projections during the first period of the SJFT. Likewise, the Uchi-komi ${ }^{\mathrm{Tot}}$ and the total projections during SJFT and FIJT were also almost perfectly significant. Indeed, these relationships between the FIJT and the SJFT confirm that FIJT is a valid measure of the physical fitness of the judoka and support the effectiveness of the present FIJT to assess the physical fitness of them.

The greater distance covered during the six repetitions of Australian shuttle test demonstrated a nearly perfect correlation with Uchi-komi ${ }^{\text {best }}$ during the FIJT. Likewise, the total distance covered during the Australian shuttle 
Correlation (r)

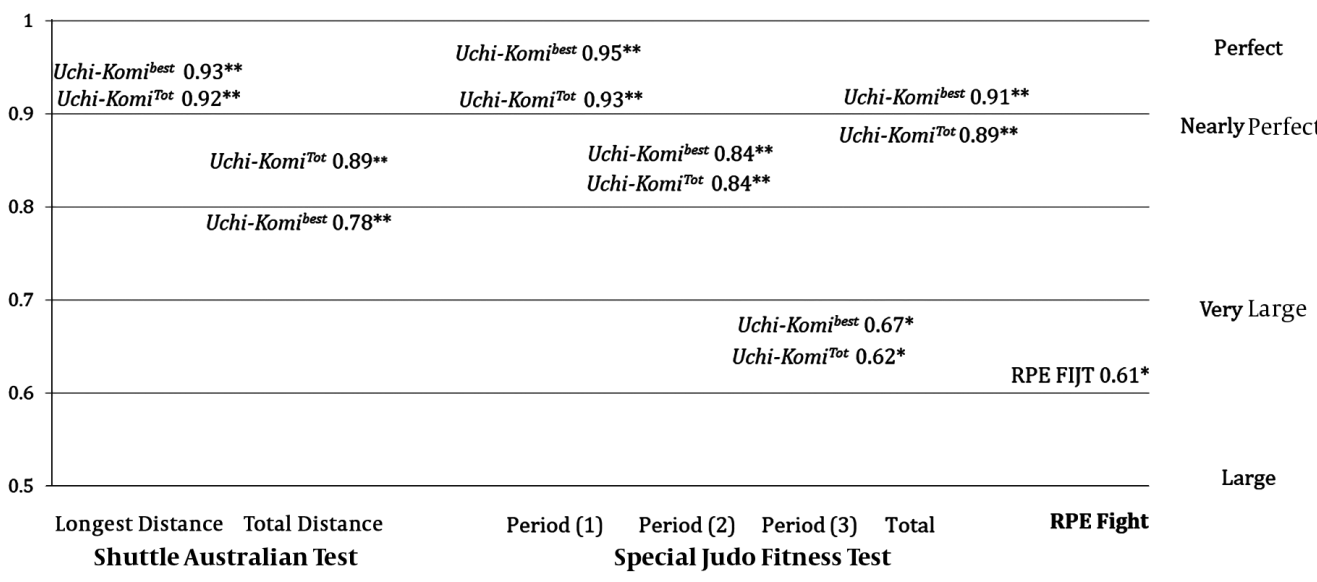

Figure 2. Pearson's product moment correlation and magnitude of the correlations between the Uchi-komi ${ }^{\text {best }}$ and Uchi-komi ${ }^{\text {Tot }}$ of the fitness intermittent judo test and the other tests. * significant correlation at $(\mathrm{P}<0.05) ;{ }^{* *}$ significant correlation at $(\mathrm{P}<0.01)$.

\begin{tabular}{|c|c|c|c|c|c|c|}
\hline Performance & Test $^{\mathrm{a}}$ & Re-Test $^{a}$ & ICC & ES & SEM & SWC \\
\hline Uchi-komi ${ }^{\text {best }}$ & $17.87 \pm 2.77$ & $18.09 \pm 2.63$ & 0.98 & 0.08 & 0.13 & 2.01 \\
\hline Uchi-komi $i^{\text {Tot }}$ & $164.04 \pm 28.91$ & $164.43 \pm 28.76$ & 0.99 & 0.01 & 0.20 & 2.96 \\
\hline
\end{tabular}

Abbreviations: ICC, intraclass correlation coefficient; ES, effect size; SEM, standard error of measurement; SWC, smallest worthwhile change.

${ }^{a}$ Values are expressed as Mean $\pm S D$.

showed also nearly perfect correlation with Uchi-komi ${ }^{\text {Tot }}$ during the FIJT. Thus, since the Australian shuttle test is widely credited to be a valid measure of anaerobic power and capacity of the athletes (21), it is safe to say that the FIJT could reproduce the actual judo exigencies and provide a valid assessment of judokas' performance. Consequently, these relationships strengthen the usefulness of the present FIJT and the data of correlation analyses between the FIJT and other physical fitness components of judo athletes such as distance running in Australian shuttle test and the number of projection in the SJFT confirm the validity of the FIJT in assessing the physical fitness of judokas. Although the reader must also be aware that the magnitude of the correlation between the FIJT and both the Australian test and the SJFT can by influenced by the specificity of the running or the use of a specific technique during the SJFT. This is particularly relevant in view of the specificity of motor skills during the FIJT. The present FIJT makes a more appropriate study to identify if there is a relationship between the number of Uchi-komi in the test and the number of drills in judo fights or the winning percentage in these fights.

The relative reliability of the FIJT showed no difference between mean test and retest scores of Uchi-komi ${ }^{\text {best }}$ and Uchi-komi ${ }^{\text {Tot }}$. The ICC values in the current FIJT were 0.98 and 0.99 for $U c h i-k o m i$ best and $U c h i-k o m i{ }^{\text {Tot }}$. It is well admitted that the ICC values of physical and physiological field tests were acceptably reliable if the ICC is over 0.80 and is considered high if it is over 0.90 (25). However, the meaningfulness of the ratio of the mean difference between test and retest performances indexes of the current FIJT were trivial (ES: 0.08 for Uchi-komi ${ }^{\text {best }}$ and 0.01 for $U$ chi-komi ${ }^{\mathrm{Tot}}$ ) which confirmed the relative reliability of the FIJT. The difference observed between the two performances indexes of the FIJT trials was certainly attributed to measurement errors. The SEM was not affected by inter-individual variability but in association with ICC (25). However, the small SEM observed in our data ( 0.13 and 0.20$)$ were in the same range of acceptable values of the SEM reported in physical and physiological field tests $(25,26)$ and strengthened the reliability of the FIJT. Accordingly, the high ICC value, the low ES, the small SEM values support the reliability of the FIJT. To establish the usefulness of the FIJT, we calculated the SWC in performance defined as the minimal individual change that can be interpreted as real with an acceptable probability level $(25,26)$. In this context, it is important for coaches administering this test to know that a change of 2 and $4 U$ Chi-komi in Uchi-komi ${ }^{\text {best }}$ and Uchi-komi ${ }^{\text {Tot }}$ respectively has occurred. For example, after a training program with male judoka, it is necessary that the change in per- 
formance highlights improvements and exceeds measurement error.

\subsection{Conclusions}

The ability to repeat intermittent maximal efforts with basic judo movement patterns and the full technical execution are decisive requirements of judo performance. The FIJT is a valid and reliable measurement of the fitness of judoka. It could be recommended for fitness assessing and training of judo athletes in the quest to enhance their performance. Further studies are necessary to investigate the sensitivity of the FIJT. The lack of physiological measurements during the FIJT and the judo fight, which determine the objective relationship between them, could be considered as a limitation of the present study. Sports scientists, strength conditioning, and judo coaches could routinely use the FIJT for both assessing and training judokas to optimize their performance. It could be also used to control the effect of training program and to monitor the physical fitness of judokas, especially with the presence of SWC of values. In fact, it is important that the Kumi-kata, Nagekomi, Ne-waza and Uchi-komi remain the same throughout the different FIJT's since the choice of the technique could influence performance.

\section{References}

1. Miarka B, Cury R, Julianetti R, Battazza R, Julio UF, Calmet M, et al. A comparison of time-motion and technical-tactical variables between age groups of female judo matches. J Sports Sci. 2014;32(16):1529-38. doi: 10.1080/02640414.2014.903335. [PubMed: 24720553].

2. Miarka B, Panissa VL, Julio UF, Del Vecchio FB, Calmet M, Franchini E. A comparison of time-motion performance between age groups in judo matches. J Sports Sci. 2012;30(9):899-905. doi: 10.1080/02640414.2012.679675. [PubMed: 22530748].

3. Blais L, Trilles F, Lacouture P. Three-dimensional joint dynamics and energy expenditure during the execution of a judo throwing technique (Morote Seoi Nage). J Sports Sci. 2007;25(11):1211-20. doi: 10.1080/02640410600982204. [PubMed: 17654233].

4. Franchini E, Del Vecchio FB, Matsushigue KA, Artioli GG. Physiological profiles of elite judo athletes. Sports Med. 2011;41(2):147-66. doi: 10.2165/11538580-000000000-00000. [PubMed: 21244106].

5. Franchini E, Yuri Takito M, Yuzo Nakamura F, Ayumi Matsushigue K, Peduti Dal'Molin Kiss MA. Effects of recovery type after a judo combat on blood lactate removal and on performance in an intermittent anaerobic task. J Sports Med Phys Fitness. 2003;43(4):424-31. [PubMed: 14767401].

6. Serrano MA, Salvador A, Gonzalez-Bono E, Sanchís C, Suay F. Relationships between recall of perceived exertion and blood lactate concentration in a judo competition. Percept Mot Skills. 2016;92(3_suppl):1139-48. doi:10.2466/pms.2001.92.3c.1139.

7. Santos L, Gonzalez V, Iscar M, Brime JI, Fernandez-Rio J, Rodriguez B, et al. Physiological response of high-level female judokas measured through laboratory and field tests. Retesting the validity of the Santos test. J Sports Med Phys Fitness. 2012;52(3):237-44. [PubMed: 22648461].

8. Franchini E, Nunes AV, Moraes JM, Del Vecchio FB. Physical fitness and anthropometrical profile of the Brazilian male judo team.JPhysiol Anthropol. 2007;26(2):59-67. [PubMed: 17435345].

9. Almansba R, Franchini E, Sterkowicz S. [Uchi-komi with load, a physiological approach of a new test specific to judo]. Science \& Sports. 2007;22(5):216-23. French. doi:10.1016/j.scispo.2007.06.006.

10. Santos L, Gonzalez V, Iscar M, Brime JI, Fernandez-Rio J, Egocheaga $\mathrm{J}$, et al. A new individual and specific test to determine the aerobic-anaerobic transition zone (Santos Test) in competitive judokas. J Strength Cond Res. 2010;24(9):2419-28. doi 10.1519/JSC.0b013e3181e34774. [PubMed: 20802284].

11. Thomas PH, Goubault C, Beau C, Brandet JP. [Judo evaluation test, derived from the Leger-Mercier test]. Medicine du Sport. 1989;63:286-8. French.

12. Sterkowicz S. [Special fitness test in judo]. Antropomotoryka. 1995;12:29-44. Polish.

13. Franchini E, Del Vecchio FB, Sterkowicz S. A special judo fitness test classificatory table. Arch budo. 2009;5:127-9.

14. Azevedo PH, Drigo AJ, Carvalho MC, Oliveira JC, Nunes JE, Baldissera $\mathrm{V}$, et al. Determination of judo endurance performance using the uchi - komi technique and an adapted lactate minimum test. J Sports Sci Med. 2007;6(CSSI-2):10-4. [PubMed: 24198697]. [PubMed Central: PMC3809042].

15. Del Vecchio FB, Dimare M, Franchini E, Schaun GZ. Physical fitness and maximum number of all-out hikidashi uchi-komi in judo practitioners. Med Dello Sport. 2014;67(3):383-96.

16. Tavra M, Franchini E, Krstulovic S. Discriminant and factorial validity of judo-specific tests in female athletes. Arch Budo. 2016;12:93-9.

17. Baudry S, Roux P. Specific circuit training in young judokas: effects of rest duration. Res Q Exerc Sport. 2009;80(2):146-52. doi: 10.1080/02701367.2009.10599548. [PubMed: 19650379].

18. Franchini E, Panissa VL, Julio UF. Physiological and performance responses to intermittent Uchi-komi in Judo. J Strength Cond Res. 2013;27(4):1147-55. doi: 10.1519/JSC.0b013e3182606d27. [PubMed: 22692119].

19. Franchini E, Brito CJ, Fukuda DH, Artioli GG. The physiology of judospecific training modalities. J Strength Cond Res. 2014;28(5):1474-81. doi: 10.1519/JSC.0000000000000281. [PubMed: 24149757].

20. Cazorla G, Boussaidi L, Godemet M, editors. . Proceedings of the Medical Congress of the French Rugby Federation: Rugby, Shoulder, Knee, Spine, Physiology. Lyon, France. Deed of Medical Congress of French Federation of Rugby; 2004. p. 435-56. French.

21. Paillard T. [Optimization of sports performance in judo]. Bruxelles: de Boeck Superieur; 2010. French.

22. Borg G, Hassmen P, Lagerstrom M. Perceived exertion related to heart rate and blood lactate during arm and leg exercise. Eur J Appl Physiol Occup Physiol.1987;56(6):679-85. doi:10.1007/bf00424810.

23. Hopkins WG. How to interpret changes in an athletic performance test. Sportscience. 2004;8(1):1-7.

24. Batterham AM, Hopkins WG. Making meaningful inferences about magnitudes. Int J Sports Physiol Perform. 2006;1(1):50-7. [PubMed: 19114737].

25. Atkinson G, Nevill AM. Statistical methods for assessing measurement error (reliability) in variables relevant to sports medicine. Sports Med.1998;26(4):217-38. [PubMed: 9820922].

26. Hopkins WG, Marshall SW, Batterham AM, Hanin J. Progressive statistics for studies in sports medicine and exercise science. Med Sci Sports Exerc. 2009;41(1):3-13. doi: 10.1249/MSS.0b013e31818cb278. [PubMed: 19092709]. 